\title{
Modeling Soil Deformation during Permafrost Thawing
}

\author{
A.V. Vasyukov ${ }^{a *}$ A.S. Ermakov ${ }^{\text {b }}$ \\ ${ }^{\mathrm{a}, \mathrm{b}}$ Keldysch Institute of Applied Mathematics RAS, Moscow 125047, Russia \\ ${ }^{a}$ *Corresponding author’s e-mail: vasyukov@gmail.com
}

Article History: Received: 10 November 2020; Revised 12 January 2021 Accepted: 27 January 2021; Published online: 5 April 2021

\begin{abstract}
The active development of the northern latitudes leads to the fact that a significant number of sources of technogenic heating arise in the permafrost regions. This shifts the existing thermal equilibrium and leads to gradual thawing of the frozen phase in the medium. This process can significantly reduce the strength of the geological massif and lead to subsidence of the soil surface, depletion of the bearing capacity of the soil, and eventually to the occurrence of landslides and sinkholes. This paper is devoted to the numerical modeling of partially melted medium in the permafrost region. The paper covers mechanical processes in the soil under static gravitational load, taking phase composition space distribution as the input. The multiphase medium is described using Biot model with the effective parameters for a composite solid phase and separate parameters for a fluid phase. The numerical solution is obtained using finite element method that is also presented in the paper. Calculation results for several two dimensional problem statements are presented and discussed in the paper
\end{abstract}

Keywords: Permafrost, melting, Biot model, porous medium, multiphase medium, static load, finite element method, computer simulation

\section{Introduction}

A significant number of modern oil and gas fields are located in permafrost regions. Extraction of deposits in such conditions is associated with significant risks due to the thawing of permafrost. Potentical risks are caused by the fact that thawing of the frozen phase in the medium changes the mechanical properties and the residual strength of the geological massif that may lead to the occurrence of landslides and sinkholes.

This research targets the problem of mathematical modeling of partially melted medium in the permafrost region. This research concentrates on static gravitational load only, leaving long-term geological processes and dynamical and vibrational processes aside. This research does not cover thermal processes in the soil, taking the space distribution of the phases as the input from third-party models. The medium is taken to be porous, consisting of a solid skeleton and a fluid saturating it. Due to the different time scales of the thermal and mechanical processes, the latter can be solved with respect to the fixed saturation distribution pattern.

The classical Gassmann model can be used to describe such a multiphase medium, taking the saturation into account [1]. This approach is not the only one possible. Another common model is the Bio model [2]. This model has gained significant acceptance as computing resources for performing calculations have become more widely available. Calculations based on the Biot model are widely used for various problems and show good agreement with experimental data [3]. With regard to the tasks related to the Arctic region, it should be noted that this model was successfully used to describe snow and ice formations [4,5]. What is extremely important, the method of determining Biot's coefficient, which is one of the key poroelastic parameters of a rock, was presented in [6], and this fact allows to link calculations and field observations.

Another possible mathematical model that can be used to describe the behavior of a porous medium is the classical Dorovsky model [7]. Unlike the Bio model, it is described by three elastic parameters instead of four. In the paper [8] simulation results were compared with the Bio-Johnson theory. Both models showed excellent agreement with each other; however, in order to accurately depict the dependence of Stoneley waves on a frequency that can be measured experimentally, special corrections may be required. It was also applied to the modeling of a well with a clay crust between the borehole fluid and the porous surrounding formation [9].

Relatively new approach for modeling complex fractured and saturated solid medium is using layered and block media with nonlinear contact conditions [10,11]. The paper [12] demonstrated that this approach can be used to take into account fluid saturation of bottom sediments in marine seismic survey. 
This paper is organized as follows: Section 2 presents the mathematical model and the numerical method that were used for this study. The numerical results are presented in Section 3. Section 4 provides the discussion of the results, and Section 5 concludes the paper.

\section{Mathematical Model and Numerical Method}

This paper describes thawing permafrost as the porous multiphase medium. The notations used for the medium are $\boldsymbol{m}$ for the porosity and $\boldsymbol{s}_{\boldsymbol{v}}$ for the fraction of melted phase. Taken this notation into account one can write for the volume $\boldsymbol{\delta} \boldsymbol{V}$ of the medium:

$$
\delta \mathrm{V}=(1-\mathrm{m}) \delta \mathrm{V}+\mathrm{m} \mathrm{s}_{\mathrm{v}} \delta \mathrm{V}+\mathrm{m}\left(1-\mathrm{s}_{\mathrm{v}}\right) \delta \mathrm{V}
$$

The overall volume of the medium consists from the following phases:

- $\quad(1-\mathrm{m}) \delta \mathrm{V}-$ the volume of soil skeleton,

- $\quad \mathrm{m} \mathrm{s}_{v} \delta \mathrm{V}-$ the volume of thawed phase (water),

- $\quad \mathrm{m}\left(1-\mathrm{s}_{\mathrm{v}}\right) \delta \mathrm{V}-$ the volume of frozen phase (ice).

In this work, splitting into physical processes is used - thermal and mechanical processes are considered separately. First, the thawing of frozen phase and the changes in the phase composition of the medium caused by it are considered. The resulting dependencies are used as input data for calculating deformations.

The solid skeleton of the soil and the ice in the solid phase are described as elastic media, and melted water is described as a liquid that perceives hydrostatic loads, but does not resist shear loads. This paper uses Biot model [2] to describe the multiphase medium with the effective parameters for a composite solid phase and separate parameters for a fluid phase. The model is described by the following equations:

$$
\begin{aligned}
& \sigma_{x x}=(\lambda+2 \mu) \frac{\partial u_{x}}{\partial x}+\lambda \frac{\partial u_{y}}{\partial y}+q\left(\frac{\partial w_{x}}{\partial x}+\frac{\partial w_{y}}{\partial y}\right) \\
& \sigma_{y y}=\lambda \frac{\partial u_{x}}{\partial x}+(\lambda+2 \mu) \frac{\partial u_{y}}{\partial y}+q\left(\frac{\partial w_{x}}{\partial x}+\frac{\partial w_{y}}{\partial y}\right) \\
& \sigma_{x y}=\mu\left(\frac{\partial u_{x}}{\partial y}+\frac{\partial u_{y}}{\partial x}\right) \\
& -p=q\left(\frac{\partial u_{x}}{\partial x}+\frac{\partial u_{y}}{\partial y}\right)+Q\left(\frac{\partial w_{x}}{\partial x}+\frac{\partial w_{y}}{\partial y}\right)
\end{aligned}
$$

where: $\boldsymbol{\sigma}_{i j}$ is a stress tensor related to the solid phase, $\boldsymbol{p}$ is a pressure in the fluid, $\boldsymbol{u}_{\boldsymbol{i}}$ and $\boldsymbol{w}_{\boldsymbol{i}}$ are displacement vectors for solid and fluid phases respectively.

Mechanical properties of the solid are described using Lame parameters $\lambda$ and $\boldsymbol{\mu}$, properties of the fluid are characterized by parameters $\boldsymbol{q}$ and $\boldsymbol{Q}$.

Finite element method is used to solve static problem. The classical approach described in [13] is used to construct the method.

For the numerical solution the equations are presented in matrix form:

$\tau=\boldsymbol{D} \cdot \boldsymbol{\varepsilon}$

In this notation stress vector $\boldsymbol{\tau}$ and deformation vector $\boldsymbol{\varepsilon}$ are given as follows:

$$
\begin{aligned}
& \boldsymbol{\tau}=\left\{\sigma_{\mathrm{xx}}, \sigma_{\mathrm{yy}}, \sigma_{\mathrm{xy}}, \mathrm{p}\right\} \\
& \boldsymbol{\varepsilon}=\left\{\varepsilon_{x x}, \varepsilon_{y y}, \varepsilon_{x y}, \zeta\right\}
\end{aligned}
$$

The relation between stresses and deformations is given by the matrix $\boldsymbol{D}$ :

$\boldsymbol{D}=\left(\begin{array}{cccc}\lambda+2 \mu & \lambda & 0 & q \\ \lambda & \lambda+2 \mu & 0 & q \\ 0 & 0 & \mu & 0 \\ q & q & 0 & Q\end{array}\right)$

Taken these notations into account, the implementation of the numerical method directly follows the scheme described in [14].

\section{Numerical results}

Several problem statements were calculated using the described mathematical model and numerical method. 
The first series of calculations considered a layered structure in a gravity field. The Figure 1a shows the geometry of the computational domain. A square area with a side of 2000 meters is considered. The upper and lower layers are 800 meters thick. The middle layer is 400 meters thick. The figure also shows a triangular mesh built for this area. In the calculation, the lower boundary of the area is rigidly fixed along both axes, which corresponds to the presence of a rocky base. The rest of the boundaries of the computational domain are taken free.

The upper and lower soil layers in this calculation were assumed to be relatively rigid and not subject to melting processes. Their mechanical parameters: density $2100 \mathrm{~kg} / \mathrm{m}^{3}$, Young's modulus $3.6 \cdot 10^{10} \mathrm{~Pa}$, Poisson's ratio 0.31 . The middle layer corresponds to the melted permafrost, in it the porosity is taken equal to 0.3 , all the liquid in the pores has melted.

The Figure 1b shows the geometry of the computational domain after the melting. The thin black frame in the figure indicates the initial shape of the computational area. It can be seen that the overall movement of the surface is significant. The Figure 2 shows the distribution of deformations in the medium. It can be seen from the Figure 2a that horizontal deformations are localized in the areas at the edges of the layer subject to melting. The vertical component of deformations is presented on the Figure $\mathbf{2} \mathbf{b}$ and is also localized in the middle layer, since as a result of melting it becomes mechanically less rigid.

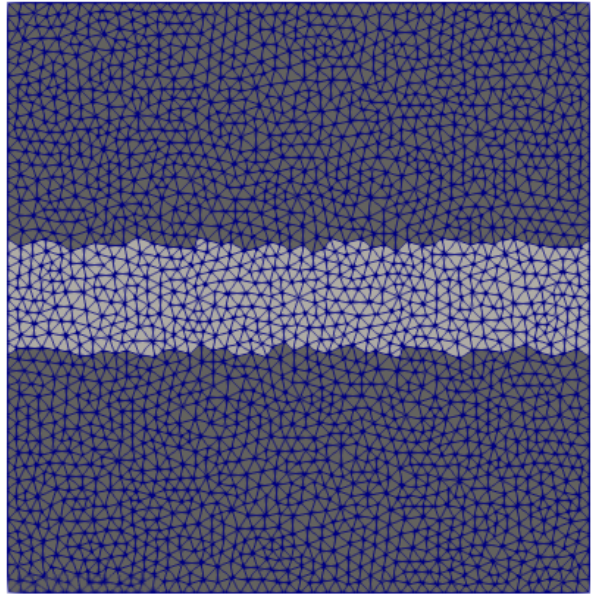

(a) Initial state

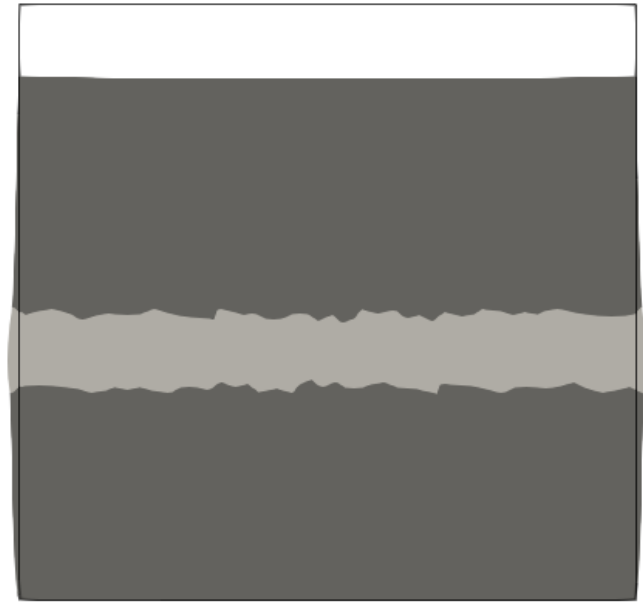

(b) Final state

Figure 1 Sandwich structure: geometry and rheology.

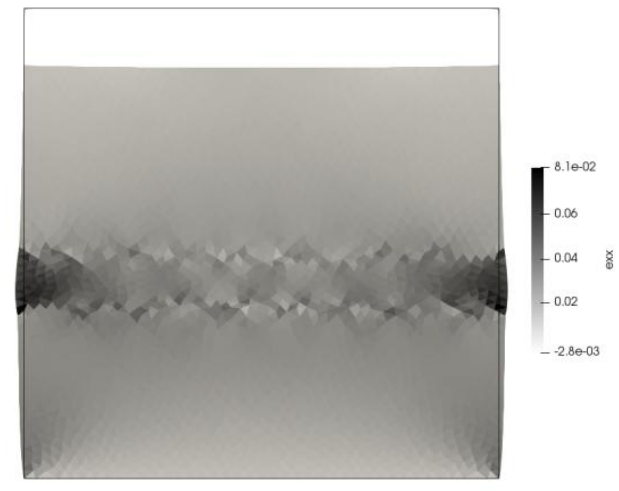

(a) $\mathrm{e}_{\mathrm{xx}}$ deformation

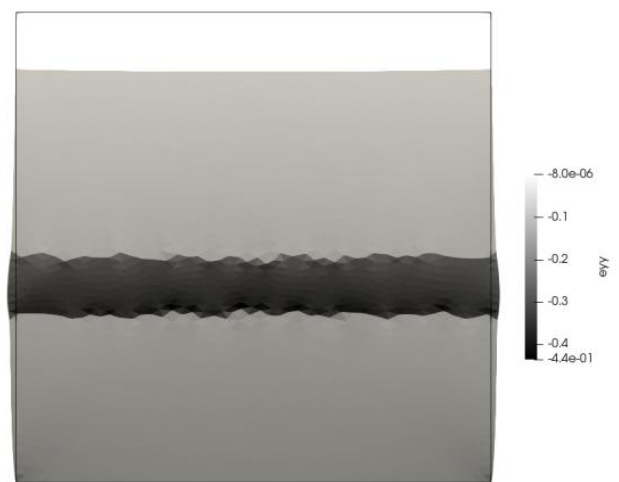

(b) $\mathrm{e}_{\mathrm{yy}}$ deformation

Figure 2 Sandwich structure: deformations.

The second series of calculations considers the melting of a large inclusion inside the soil massif. The Figure 3 shows the general geometry of the computational domain - the initial one with a frozen inclusion (Figure 3a) and the final one with a melted inclusion (Figure 3b). The size of the calculation area is $2000 \times 2000 \mathrm{~m}$. The linear size of the inclusion is $400 \mathrm{~m}$. The mechanical characteristics of the soil in the initial state and after the melting of the inclusion are the same as in the calculations of the layered structure. 
The boundary conditions in this calculation should be emphasized -- the lower boundary is fixed along both axes, and the side faces are fixed only in the horizontal direction, but can move in the vertical direction. This formulation of the boundary conditions makes it possible to obtain uniform stress and strain fields in the horizontal direction for the initial geometry (before the melting of the inclusion). The distribution of all components of the stress tensor before the melting of the inclusion is shown in the Figure 4. One can see homogeneous fields of the normal tensor components and almost complete absence of shear stresses.

After the inclusion melts, the stress distribution in the medium changes significantly. The distributions of all tensor components are shown in the Figure 5. Diagonal patterns are noticeable, oriented from the edges of the inclusion. Some change in the values of the vertical and horizontal normal components can be seen, which is associated with a change in the effective mechanical properties of the melted inclusion. The most significant change in shear stresses (Figure 5c) -- they appeared after the inclusion melted, which is associated with the redistribution of loads into the base and upper part of the geological massif.

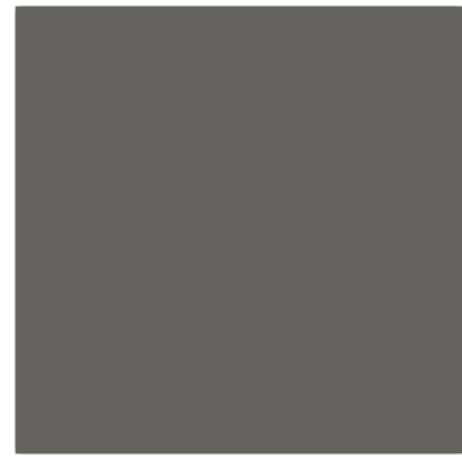

(a) Initial state

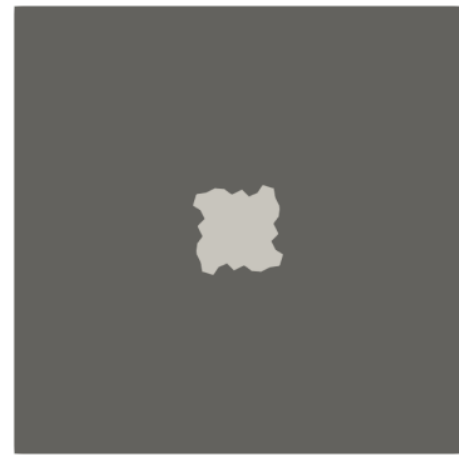

(b) Melted state

Figure 3 Inclusion: geometry and rheology.

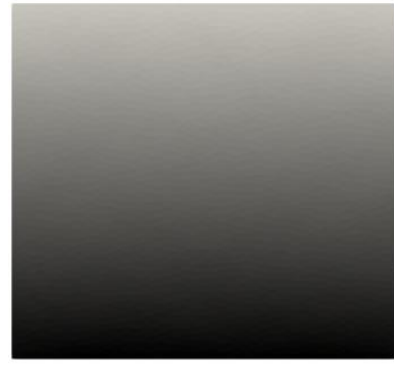

(a) $\sigma_{y y}$

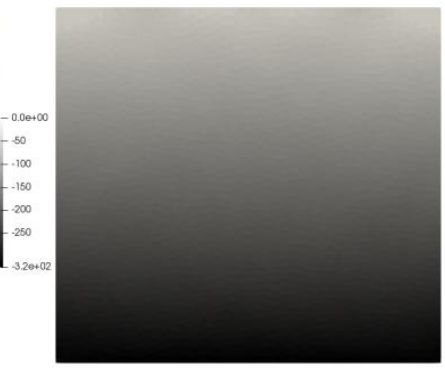

(b) $\sigma_{x x}$

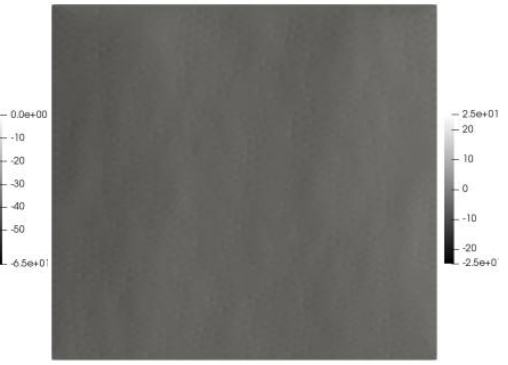

(c) $\sigma_{\mathrm{xy}}$

Figure 4 Frozen inclusion: stress distribution.

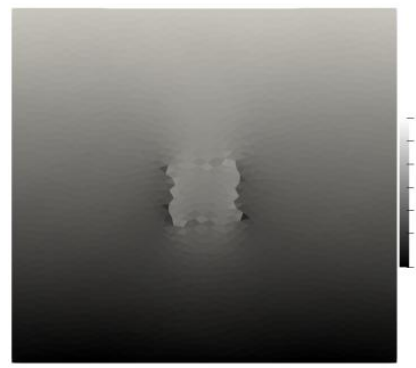

(a) $\sigma_{y y}$

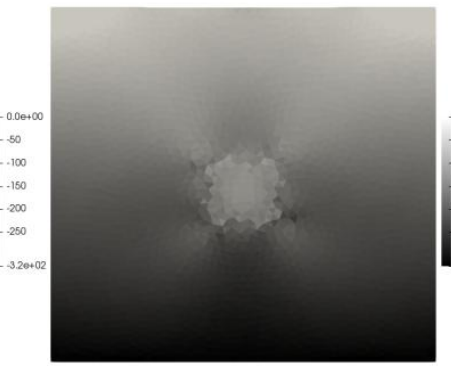

(b) $\sigma_{\mathrm{xx}}$

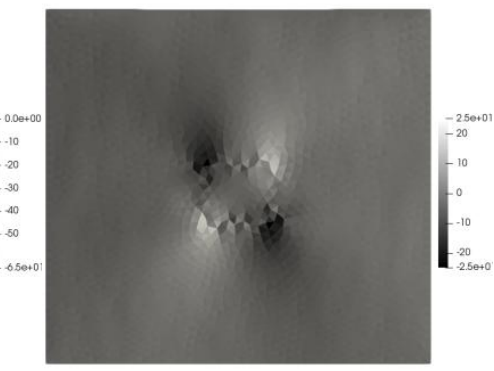

(c) $\sigma_{x y}$

Figure 5 Melted inclusion: stress distribution. 


\section{Discussion}

The numerical results obtained in this paper are consistent with expectations based on general considerations, engineering intuition and field observations. The most interesting results obtained are the patterns of the redistribution of stresses in the medium during the melting of a large inclusion. It is clearly seen that this process leads to the appearance of shear components of the stress tensor. Shear stresses are of particular interest since soils do not withstand this type of load very well. The appearance of shear stress components can lead to the depletion of the bearing capacity of the soil and the subsequent occurrence of landslides, failures and other negative consequences.

Direct quantitative application of the model to assess the bearing capacity of partially melted permafrost is significantly hampered by the lack of reliable quantitative data on the strength of soils under different types of loading. However, for a specific environment, it is possible to calibrate the described model according to the data of laboratory tests of soil samples.

\section{Conclusion}

The results of calculations show that Biot model and the finite element method can be used to model the mechanical behavior of a multiphase medium that is formed during thawing of permafrost. However, the methodology described in this paper is not exhaustive and assumes significant development in the future.

It should be noted that the approach presented in this work does not consider the long-term effects of soil mechanics - soil creep, its consolidation with changes in moisture content, and the effect of these processes on long-term strength. The model used in this work is focused on calculating the deformation of a multiphase medium immediately after its thawing, and consideration of longer processes is a separate problem.

Another factor that will be important when calculating large areas is taking into account the dependence of the properties of the medium on pressure. For soils, this dependence is a known fact. Supplementing the technique described in this work with the corresponding equations is necessary when calculating soils at large depths.

These factors are supposed to be considered in separate subsequent works.

\section{Acknowledgement}

This work was supported by the Russian Science Foundation, grant no. 16-11-00100p.

\section{References}

F Gassmann. Elasticity of porous media. Vierteljahrsschrder Naturforschenden Gesselschaft. 1951; 96, 1-23.

MA Biot. Theory of propagation of elastic waves in a fluid-saturated porous solid. I. Low-frequency range. J. Acoust. Soc. Am. 1956; 28 (2), 168-178.

KW Winkler, HL Liu and DL Johnson. Permeability and borehole Stoneley waves: Comparison between experiment and theory. Geophysics, 1989; 54 (1), 66-75.

R Sidler. A porosity-based Biot model for acoustic waves in snow. Journal of Glaciology. 2015; 61 (228), 789 798.

A Capelli, JC Kapil, I Reiweger, D Or and J Schweizer. Speed and attenuation of acoustic waves in snow: Laboratory experiments and modeling with Biot's theory. Cold Reg. Sci. Technol. 2016; 125, 1-11.

J He, Z Rui and K Ling. A new method to determine Biot's coefficients of Bakken samples. J. Nat. Gas. Sci. Eng. 2016; 35 (A), 259-264.

VN Dorovsky. Continual theory of filtration (in Russian). Sov. Geol. Geophys. 1989; 30 (7), 39-45.

VN Dorovsky, YuV Perepechko and AI Fedorov. Stoneley waves in the Biot-Johnson and continuum filtration theories (in Russian). Rus. Geol. Geophys. 2012; 53 (5), 621-632.

AV Sinev, EI Romensky and VN Dorovsky. Effects of a mudcake on Stoneley waves in a fluid-filled porous formation around a borehole. Russian Geology and Geophysics. 2012; 53 (8), 823-828. https://doi.org/10.1016/j.rgg.2012.06.009

IS Nikitin, NG Burago, VI Golubev and AD Nikitin. Methods for Calculating the Dynamics of Layered and Block Media with Nonlinear Contact Conditions. In: L Jain, M Favorskaya, I Nikitin, D Reviznikov (Eds.). Advances in Theory and Practice of Computational Mechanics. Springer, Singapore, 2020. https://doi.org/10.1007/978-981-15-2600-8_13

IS Nikitin, NG Burago, VI Golubev and AD Nikitin. Mathematical modeling of the dynamics of layered and block media with nonlinear contact conditions on supercomputers. J. Phys.: Conf. Ser. 2019; 1392 (1), 012057.

VI Golubev, AV Shevchenko and IB Petrov. Taking into Account Fluid Saturation of Bottom Sediments in $\begin{array}{llllll}\text { Marine Seismic Survey. Doklady Mathematics. 2019; } 100 & \text { (2), 488-490. }\end{array}$ https://doi.org/10.1134/S1064562419050107 
OC Zienkiewicz and RL Taylor. Finite Element Method: Volume 1. The Basis. 5th Oxford: ButterworthHeinemann, 2000.

VV Aksenov, AV Vasyukov and IB Petrov. Numerical modelling of thin anisotropic membrane under dynamic load. Aeronaut. J. 2020; 1-18. https://doi.org/10.1017/aer.2020.61 